\title{
Constraints on a strong X-ray flare in the Seyfert galaxy MCG-6-30-15
}

\author{
R. W. Goosmann ${ }^{1}$, B. Czerny ${ }^{2}$, V. Karas ${ }^{1}$, M. Dovciak ${ }^{1}$, \\ G. Ponti ${ }^{3}$, and M. Mouchet ${ }^{4}$ \\ 1 Astronomical Institute, Academy of Sciences, Boční II 1401, 14131 Prague, Czech Rep. \\ ${ }^{2}$ Copernicus Astronomical Center, Bartycka 18, 00-716 Warsaw, Poland \\ 3 Dipartimento di Astronomia, Università di Bologna, Via Ranzani 1, 40127, Bologna, Italy \\ ${ }^{4}$ Laboratoire ApC, Université Denis Diderot, 2 place Jussieu, 75251 Paris Cedex 05, France
}

\begin{abstract}
We discuss implications of a strong flare event observed in the Seyfert galaxy MCG-630-15 assuming that the emission is due to localized magnetic reconnection. We conduct detailed radiative transfer modeling of the reprocessed radiation for a primary source that is elevated above the disk. The model includes relativistic effects and Keplerian motion around the black hole. We show that for such a model setup the observed time-modulation must be intrinsic to the primary source. Using a simple analytical model we then investigate time delays between hard and soft X-rays during the flare. The model considers an intrinsic delay between primary and reprocessed radiation, which measures the geometrical distance of the flare source to the reprocessing sites. The observed time delays are well reproduced if one assumes that the reprocessing happens in magnetically confined, cold clouds.
\end{abstract}

Keywords. galaxies: active, galaxies: Seyfert, X-rays: individual (MCG-6-30-15)

The Seyfert galaxy MCG-6-30-15 was observed for $95 \mathrm{ksec}$ with XMM-Newton in the year 2000 (Wilms et al. 2001). The X-ray lightcurve of this observation reveals a strong flare event of which Ponti et al. (2004) conducted a detailed analysis. In this proceedings note we discuss the possibility that the flare is produced by localized magnetic reconnection and we constrain some details of such a flare setup.

\section{Model lightcurves for an elevated flare source}

We imagine that the strong flare in MCG-6-30-15 originates in a compact reconnection site elevated to a height $H$ above the surface of the accretion disk. The radiation from this primary source partly shines toward the disk and creates a hot spot. The distance of the spot's center to the disk center is denoted by $r$. The flare is supposed to be in Keplerian co-rotation with the disk and we assume that the primary illumination sets on and fades out instantaneously. The irradiation of the disk then evolves across the hot spot, starting from the spot center and progressing toward the border. Therefore we expect the lightcurve of the reprocessed radiation to be curved even if the time evolution of the primary is box-shaped.

We want to model the exact shape of the lightcurve expected from such a flare setup at different orbital phases of the disk. We first conduct detailed local radiative transfer computations. The varying intensity of the irradiation across the hot spot is taken into account as well as the angular dependence of the reprocessed emission. The vertical structure of the disk is assumed to remain in the same hydrostatic equilibrium as before the onset of the flare. The profile is computed with an extended version of the code described in Różańska et al. (2002). The local spectra across the spot are then computed by the codes TitAN and NOAR (Dumont et al. 2000, 2003). We assume a black hole mass 

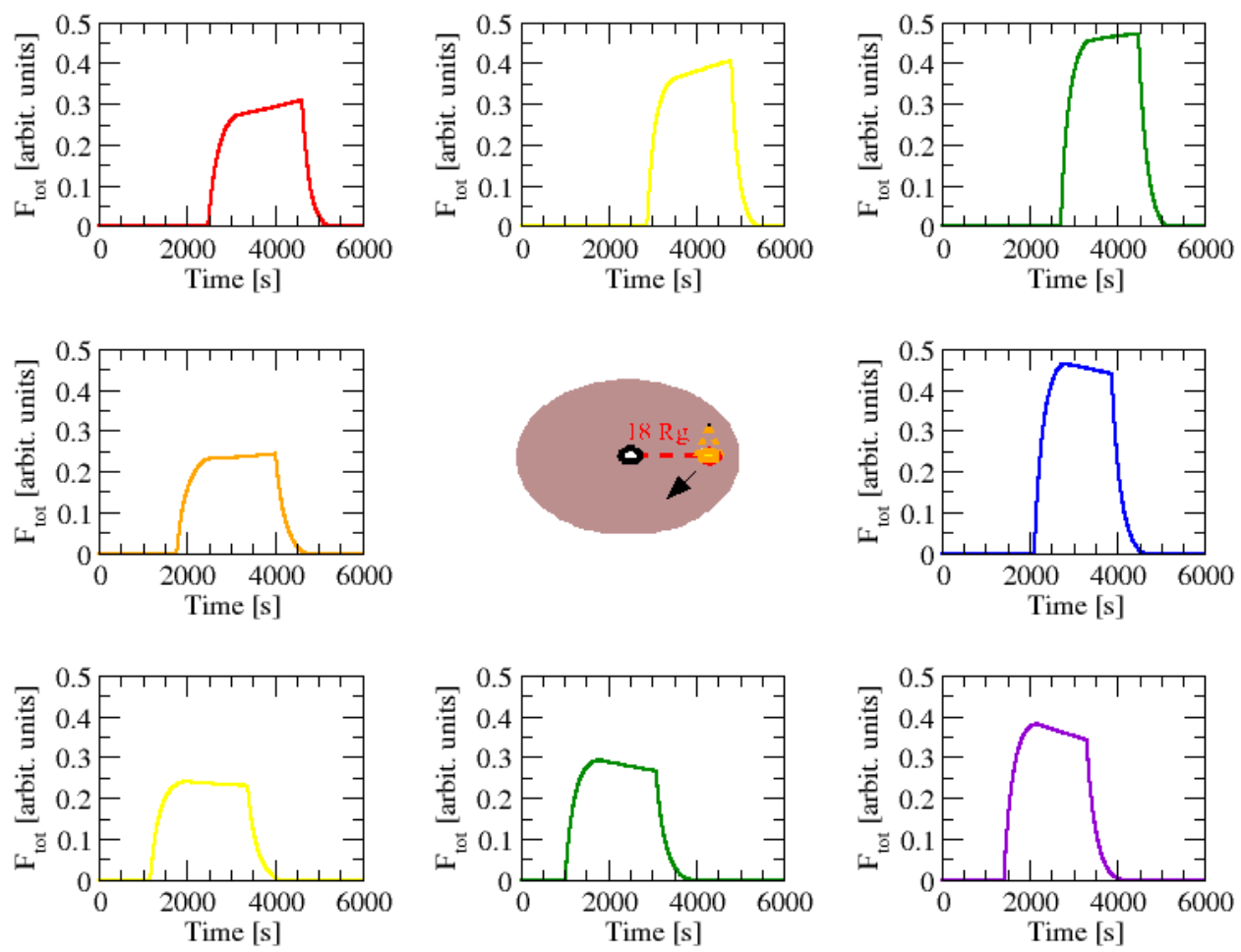

Figure 1. Lightcurves of the reprocessed spectrum integrated between $2 \mathrm{keV}$ and $10 \mathrm{keV}$ for a flare lasting $2000 \mathrm{sec}$ occurring at different orbital phases. The position of the distant observer is toward the bottom.

$M=10^{7} \mathrm{M}_{\odot}$, a disk accretion rate $\dot{m}=0.02$ (in units of the Eddington accretion rate), and $r=18 R_{\mathrm{g}}$ (with $R_{\mathrm{g}}=G M / c^{2}$ ).

Based on the local spectra, the relativistic ray-tracing code KY (Dovčiak et al. 2004) computes the time evolution of the spectrum seen by a distant observer. We set the disk inclination to $i=30^{\circ}$ and assume a Kerr parameter $a / M=0.998$ having known characteristics of MCG-6-30-15 in mind. In KY we define the time-dependent disk emission according to the subsequent illumination and fade-out of the spot from its center toward the border. The duration of this time sequence is normalized by $H$, which we constrain from the observed soft-to-hard time delay of $\sim 600 \mathrm{~s}$ (Ponti et al. 2004). We assume that this delay is entirely due to the light traveling time between the source and the disk leading to $H=7 R_{\mathrm{g}}$. The flare lasts for $2000 \mathrm{sec}$, which at $r=18 R_{\mathrm{g}}$ corresponds to $1 / 12$ of the Keplerian time scale. More details about this type of flare model are given in Goosmann (2006).

In figure 1 we show the model lightcurves obtained for flares at 8 different orbital positions. They show significant differences in shape and in normalization when comparing different orbital phases. All curves have a broadened maximum which does not match the observed, peaked shape (see Fig. 4 in Ponti et al. 2004). Thus, even if the flare is reflection-dominated, neither the geometrically evolving irradiation across the spot nor relativistic and Doppler modifications can account for the observed shape of the lightcurve. An intrinsic time evolution of the primary source is therefore required. 


\section{Modeling spectral time delays for a flare}

Ponti et al. (2004) conducted a timing analysis for the observed flare and computed cross-correlation functions and time delays between six different energy bands $\Delta E_{\mathrm{i}}$ (see their Fig. 6). We attempt to reproduce the observed time delays using the following, simple mathematical parameterization for the primary radiation $I_{\mathrm{p}}$ and the reprocessed component $I_{\mathrm{r}}$ :

$$
\begin{aligned}
I_{\mathrm{p}}(E, t) & =\mathcal{L}_{\mathrm{p}}(t) E^{-\alpha_{\mathrm{p}}}, \\
I_{\mathrm{r}}(E, t) & =N \mathcal{L}_{\mathrm{r}}\left(t_{0}, \delta, T_{\mathrm{f}}, b\right) E^{-\alpha_{\mathrm{r}}},
\end{aligned}
$$

The spectral shapes are thus represented by power laws with indexes $\alpha_{\mathrm{p}}$ and $\alpha_{\mathrm{r}}$. The time modulations $\mathcal{L}_{\mathrm{p}}(t)$ and $\mathcal{L}_{\mathrm{r}}(t)$ are defined by:

$$
\mathcal{L}_{\mathrm{p}}(t)=\frac{T_{\mathrm{f}}^{2}}{\left(t-t_{0}\right)^{2}+T_{\mathrm{f}}^{2}}, \quad \mathcal{L}_{\mathrm{r}}(t)=\frac{b^{2} T_{\mathrm{f}}^{2}}{\left[t-\left(t_{0}-\delta\right)\right]^{2}+b^{2} T_{\mathrm{f}}^{2}} .
$$

These terms account for the intrinsic change of the primary that is suggested by the modeling presented in section 1 The overall factor $N$ normalizes $I_{\mathrm{r}}$ against the primary and $\delta$ denotes an intrinsic delay between the two components. The time evolution of the reprocessing can be broadened by choosing $b>1$. This represents a spread of the light travel-time between the source and the reprocessing site. For further details on this model see Goosmann et al. (2007).

A distant observer detects the sum $I_{\mathrm{obs}}$ of $I_{\mathrm{p}}$ and $I_{\mathrm{r}}$ :

$$
I_{\mathrm{obs}}(E, t)=I_{\mathrm{p}}(E, t)+I_{\mathrm{r}}(E, t) .
$$

To investigate the time delay between the spectral response of the energy bands $\Delta E_{\mathrm{i}}$ and $\Delta E_{\mathrm{j}}$ we compute cross-correlation functions

$$
F_{\mathrm{CCF}}^{\mathrm{ij}}(\tau)=\frac{\int_{-\tau_{\max }}^{+\tau_{\max }} L_{\mathrm{i}}(t) L_{\mathrm{j}}(t-\tau) d t}{\sqrt{\int_{-\tau_{\max }}^{+\tau_{\max }} L_{\mathrm{i}}^{2}\left(t^{\prime}\right) d t^{\prime}} \times \sqrt{\int_{-\tau_{\max }}^{+\tau_{\max }} L_{\mathrm{j}}^{2}\left(t^{\prime \prime}\right) d t^{\prime \prime}}},
$$

where the lightcurve $L_{\mathrm{i}}$ belongs to the energy bin $\Delta E_{\mathrm{i}}$. We adopt here the very same method as was used in the analysis of Ponti et al. (2004). The values for $t_{0}=500 \mathrm{sec}$ and $T_{\mathrm{f}}=2200 \mathrm{sec}$ are adjusted to the flare lightcurve plotted in Fig. 4 of Ponti et al. (2004).

The spectral slope of the reprocessed component has a major impact on the obtained energy-dependent time delays. We choose a rather hard slope of $I_{\mathrm{r}}$ and set $\alpha_{\mathrm{r}}=0.1$. Such a spectral shape corresponds to the reprocessing in a medium of magnetically confined, cold clouds as analyzed by Kuncic, Celotti, \& Rees (1997). Their Fig. 2 shows reprocessed model spectra that are much harder than the primary due to multiple soft $\mathrm{X}$-ray absorption by the individual clouds. For the primary slope we set $\alpha_{\mathrm{p}}=1.3$ having in mind that for the higher flux state during the flare the spectrum should steepen (Shih, Iwasawa, \& Fabian 2002).

The model can reproduce the observed time lags for the observed flare of MCG-630-15. In Fig. 2 we show two examples of satisfactory data representation. The results account for the slight flattening of the delay curve toward higher energies. It turns out that different choices of parameters can lead to similar delay curves, as there are three free parameters involved $(N, b$, and $\delta)$ and the error bars of the data are large. To distinguish 


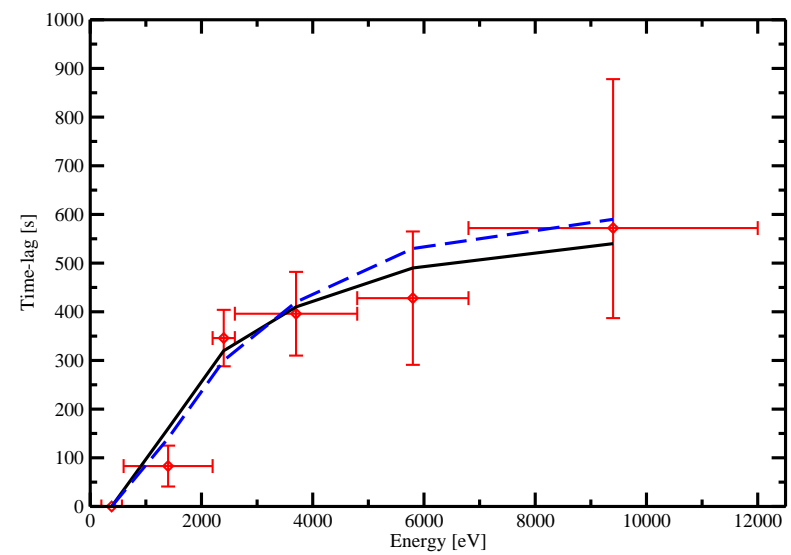

Figure 2. Best representation of the measured time-lags (red diamonds with error bars) for the flare/clumps scenario. The three curves rely on the following parameterization: $\delta=1000 \mathrm{sec}$, $b=4$ and $N=0.9$ (solid black line) and $\delta=900 \mathrm{sec}, b=3$ and $N=0.6$ (dashed blue line).

between different parameter sets some fine tuning of the delay curve's shape would be necessary. This is not meaningful with the current data.

The observed time-delay cannot be reproduced when the spectral slope of $I_{\mathrm{r}}$ is softer, i.e. adjusted to an ionized reflection scenario as proposed by Ballantyne, Vaughan, \& Fabian (2003). If the primary source is located within $\sim 10 R_{\mathrm{g}}$ of the black hole, the relative variability of the primary and the reflection component can be reproduced (Miniutti \& Fabian 2004). Such a setup also explains the high equivalent width of the iron K $\alpha$ line, which requires a relatively large amount of reflection. While these ionized reflection and light-bending models are certainly important to explain the behavior of MCG-6-30-15 on longer time-scales, the data obtained during the $2000 \mathrm{sec}$ flare period does not allow to conclude on the time evolution of the iron $\mathrm{K} \alpha$ line. From the results of our delay modeling, we therefore suggest the possibility that the strong individual flare does not originate in the inner accretion flow, where most of the X-ray energy is dissipated. We rather imagine that the flare occurred farther away from the black hole, in a region where the disk is fragmented and replaced by magnetically confined, cold clumps.

\section{Acknowledgements}

We are grateful to A.-M. Dumont and A. Różańska for their help with computing the local spectra used in this model.

\section{References}

Ballantyne D. R., Vaughan S., Fabian A. C., 2003, MNRAS, 342, 239

Dumont A.-M., Abrassart A., Collin S. 2000, A\&A, 357, 823

Dumont, A.-M., Collin, S., Paletou, F., Coupé, S., Godet, O., Pelat, D. 2003, A $\mho A, ~ 407,13$

Goosmann, R. W., Czerny, B., Karas, V., Ponti, G., Dumont, A.-M., submitted to $A \& A$

Goosmann, R. W., PhD thesis, Universität Hamburg, Germany

Kuncic, Z., Celotti, A., \& Rees, M. J. 1997, MNRAS, 284, 717

Miniutti, G., \& Fabian, A. C. 2004, $M N R A S, 349,1435$

Ponti, G., Cappi, M., Dadina, M., \& Malaguti, G. 2004, A\&A, 417, 451

Różańska A., Dumont A.-M., Czerny B., Collin S., 2002, MNRAS, 332, 799

Shih, D. C., Iwasawa, K., \& Fabian, A. C. 2002, MNRAS, 333, 687

Wilms J., Reynolds C. S., Begelman M. C., Reeves J., Molendi S. et al., 2001, MNRAS, 328, L27 